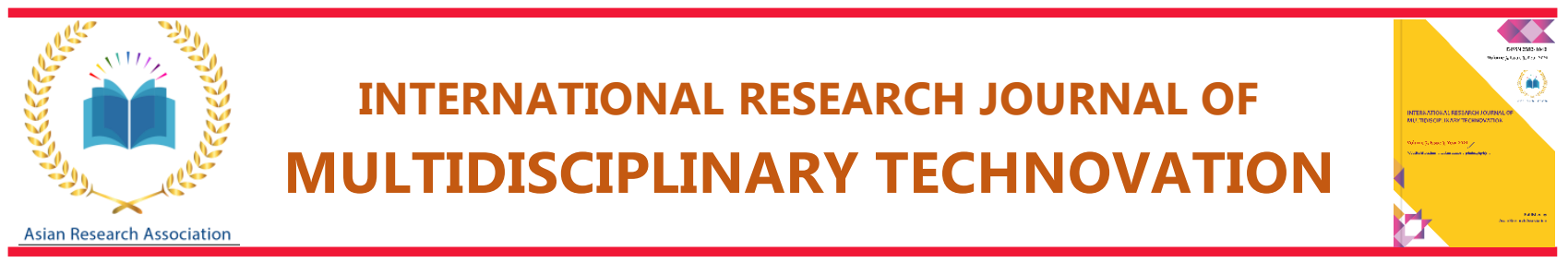

\title{
Intersecting Linear Light Strands: a Particle Explanation for the Fresnel Central Spot
}

\author{
Srin Dutt ${ }^{1,}$ * \\ ${ }^{1}$ Eyecare Centers of Florida, USA \\ ${ }^{*}$ Corresponding authors email: eyecarecenter@outlook.com \\ DOI: https://doi.org/10.54392/irjmt2213 \\ Received: 31-12-2021, Revised: 27-01-2022; Accepted: 28-01-2022, Published: 30-01-2022
}

Abstract: The Fresnel central spot in a shadow, theorized to be generated from wave interference, has given strong support for wave models of light. Using extended exposure macro imaging in various media, the shadow is shown to be more complex with multiple lines of light intersecting in the center. Using the particle concept of discrete light strands perpendicularly reflecting from a diffraction source, these observations can be more accurately explained. Another quantized aspect of light is shown where linear light strands become rings when an energy threshold is reached.

Keywords: Light, Particle concept, Energy threshold

\section{Introduction}

Descriptions of light behavior have alternated between particle and wave theory [1,2]. Strong experimental support of both models has led to a dual particle-wave theory [3-5]. The Fresnel spot, otherwise referred to as the Poisson's spot or Arago's spot [6,7], has been utilized to strongly support wave theory [8-10]. It is created by projecting light onto a solid disc which then creates a black shadow behind it where light transmission is blocked. However, a faint dot of light is visible in the center of the shadow (Figure 1).

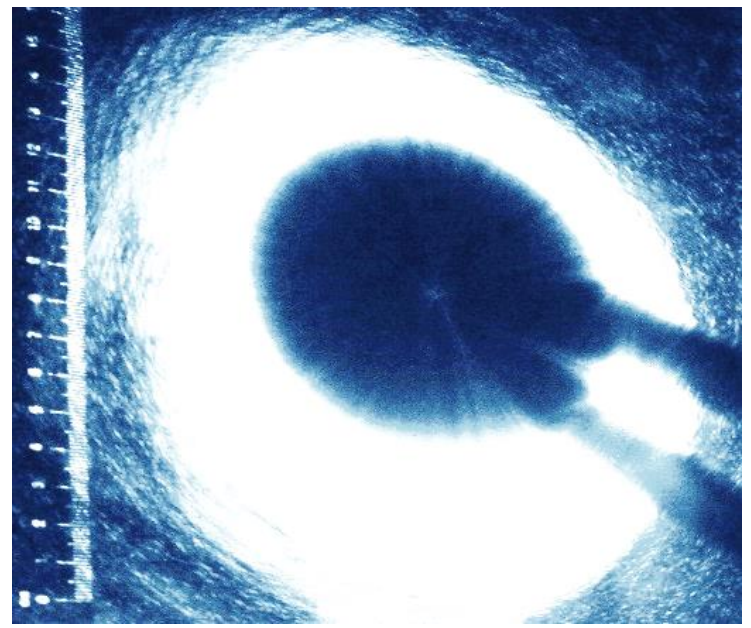

Figure 1. Classic representation of the Fresnel spot as a faint light in the center of a shadow

This is predicted from wave theory wherein new wave fronts, thought to be created at the rim of the disc, interfere with each other as they emanate forward.
Constructive interference at the equidistant center of the shadow is thought to create the central spot.

An alternative particle concept to the wave theory of light has been recently described [11]. Light interference fringe patterns have been shown to be pseudo- interference patterns in certain situations, created by discrete light strands reflecting off a diffraction source. As a substitute to wave interference explanations, the fringe patterns can be explained by these discrete light strands with clear intervening spaces that radiate outward perpendicularly when light hits an edge. Could this alternative explanation apply to the Fresnel central spot? The central spot, its surrounding shadow, and its preceding pathway in free space was analyzed to determine the validity of this conjecture.

\section{Methods}

A configuration was assembled to project light onto a solid object creating a shadow (Figure 2). A 532 $\mathrm{nm}, 5 \mathrm{mw}$ spot laser was used as a coherent light source. The light beam was transmitted through a diverging 20D lens. The expanded beam was centered over a solid disc which was held with an alligator clamp. The resultant image was isolated using an adjustable iris diaphragm to block higher intensity peripheral light outside of the shadow and allow subtle low light capture. The resulting image was projected onto a black cardstock screen. In another configuration, a solid square object was also utilized to create a shadow. 


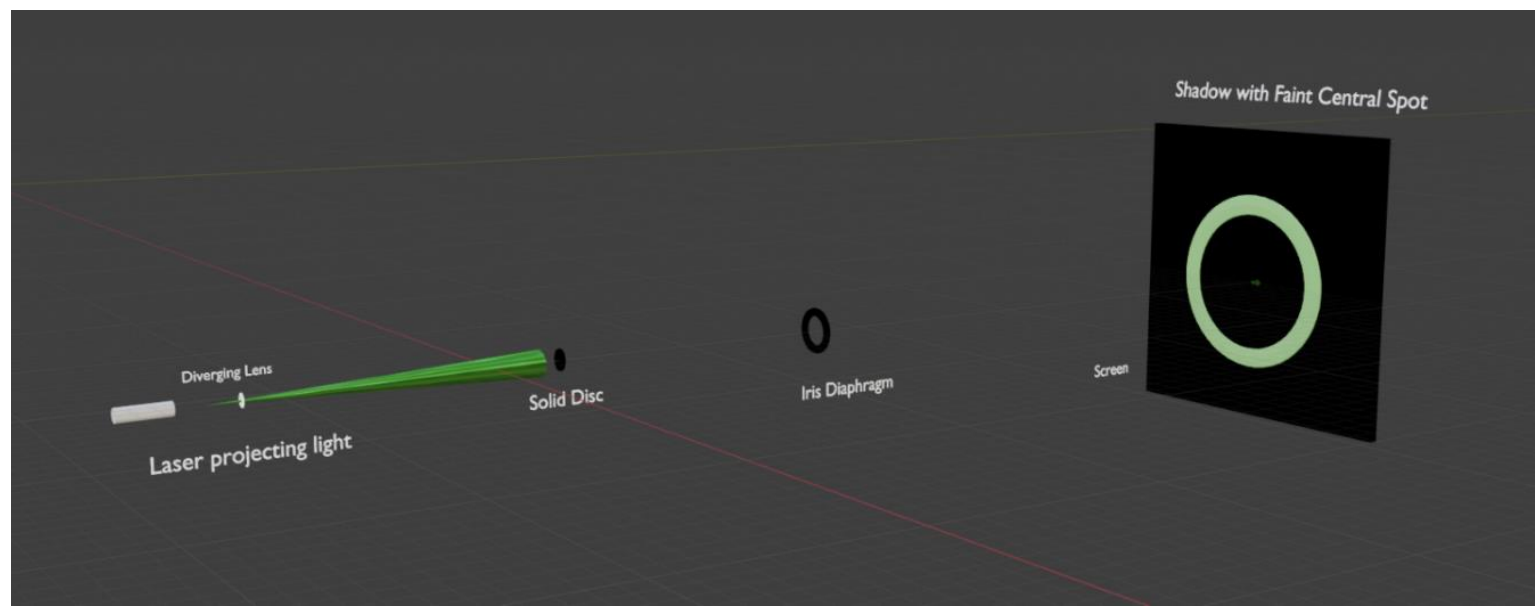

Figure 2. Light projecting onto a solid object creating a shadow and faint central spot

Rings and hollow tubes placed in free space were also used to test the screen image. Images were captured using a Lumix ZS100 digital camera with 20.1megapixel 1-inch sensor and Leica DC Vario-Elmarit Lens F2.8-5.9 using slower shutter speeds for low light extended exposure and macro imaging. Additionally, a block of transparent gelatin was utilized to view light propagating through free space prior to contacting the screen surface. Water vapor was also used as a cloud chamber to view light propagation in free space.

\section{Results}

The macro extended exposure images revealed new detail about light behavior within the shadow. Multiple individual lines of light are viewed radiating towards the center from the rim of the disc shadow (Figure 3).

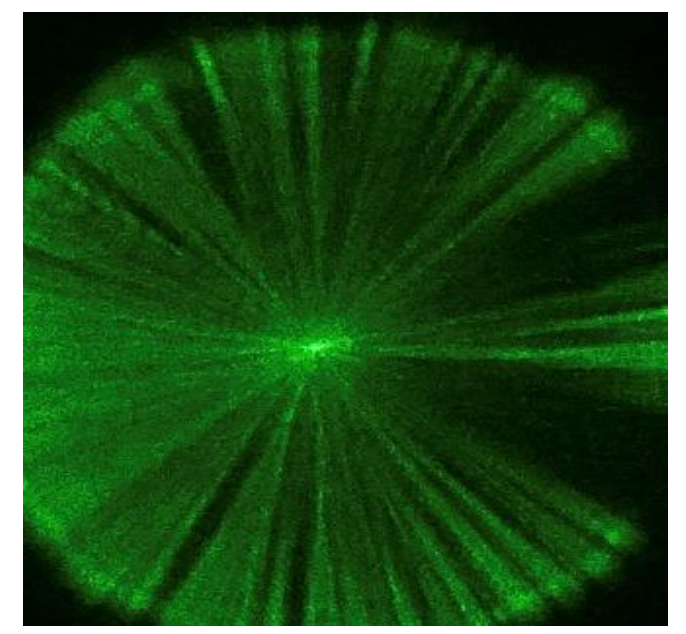

Figure 3. Multiple lines of light extending from the rim and intersecting at a central point. Some of the lines show a fringe pattern.

They can be seen intersecting at a central point as they continue past each other. As all lines pass through this central point, it is viewed as the brightest area and appears as a spot from a distance but is irregular when magnified.
If a ring is placed in front of the lines of light, a sharp corresponding shadow is created that doesn't affect adjacent areas (Figure 4). The central opening of the ring continues to show light transmission.

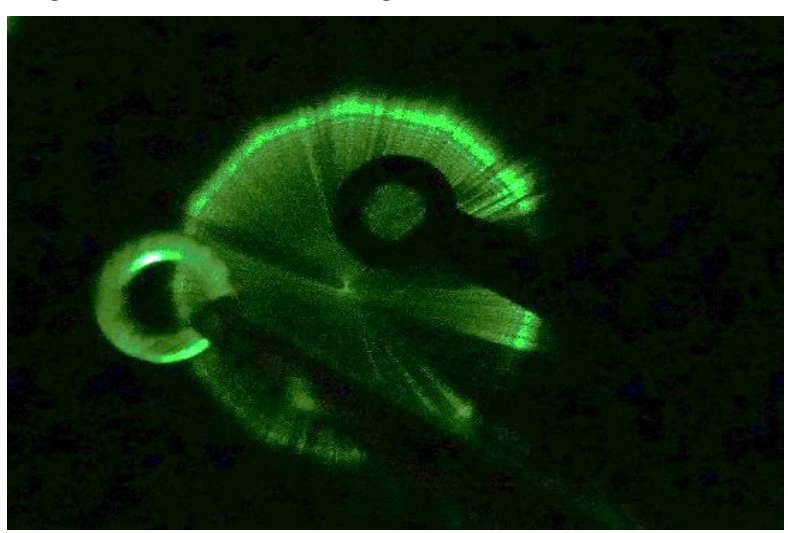

Figure 4. Ring object allowing light radiation through its central opening while sharply blocking light with its solid component

Subsequently, a small hollow tube was placed in front of the lines of light, perpendicular to the screen. This created a solid circular shadow with no light passing through its center (Figure 5). Light can be seen reflecting off the outer aspect of the tube.

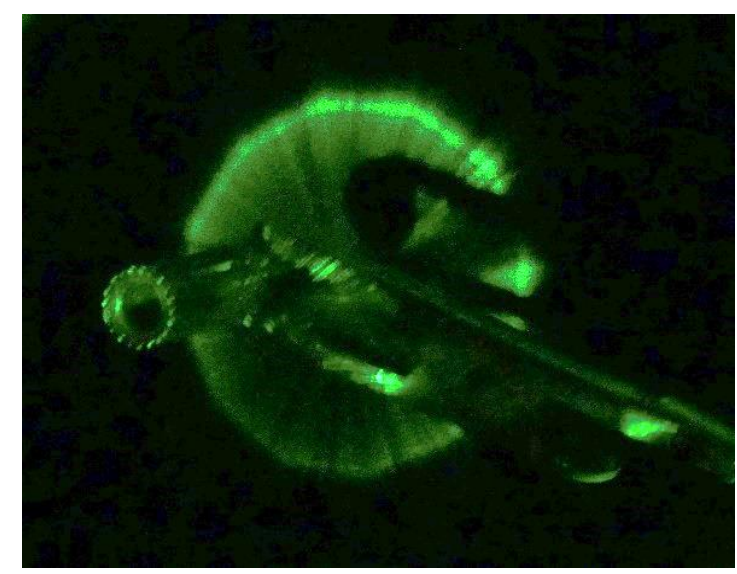

Figure 5. Hollow tube not allowing light transmission 
The alligator clamps, which were holding the disc, blocked light with its two jaws at the rim which is the point of origin of diffraction (Figure 6). The resulting blockage was distinct with sharp edges that carried to the center of the shadow and conformed to the size and shape of the jaws. If the jaws were moved, the blocked areas moved correspondingly.

If light was shown on only a portion of the disc rim, then the lines would only occur in that section (Figure 7). If most of the disc rim was left dimmed, except for a small locus, single lines with a fringe pattern would appear.

In the gelatin block, fine individual strands of light can be viewed radiating from the rim of the disc as striations (Figure 8). The brightest strands correspond to light radiating linearly from the rim of the disc to the rim of the shadow. Within the central shadow, finer, low intensity strands are seen radiating towards the center. These strands intersect in the middle of the shadow creating the pseudo-spot.

In the cloud chamber of water vapor, the cylindrical outline of the light radiation is noted (Figure 9). It is striated as it consists of individual light strands. The sharp border of two clear areas are visible traversing with the light strands and corresponding to the two jaws of the alligator clamp that blocked diffraction at the rim. Another view inside the projected light cylinder also shows the striated light strands around the periphery with distinct demarcation of the areas where the light is blocked by the clamp jaws. Faint strands are noted crossing towards the center creating the central spot.

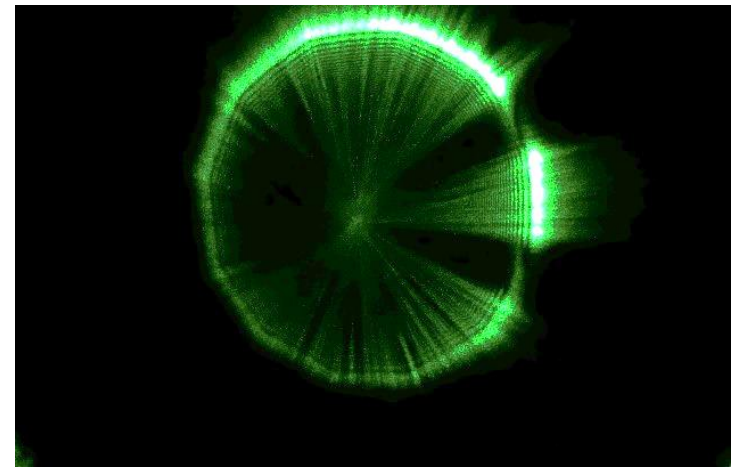

Figure 6. Clamp jaws blocking light transmission at source of diffraction, creating sharply demarcated clear areas
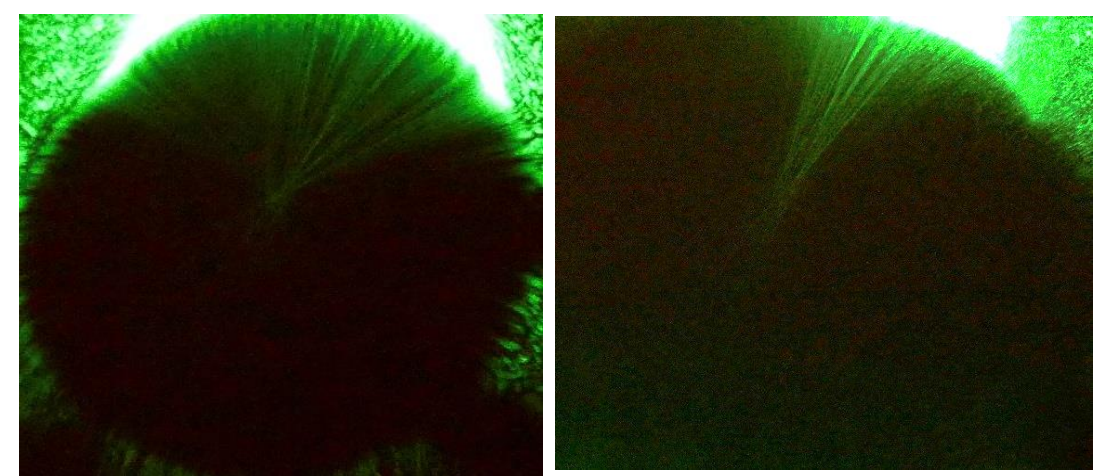

Figure 7. Partial illumination of rim creating distinct projected lines localized to that sector

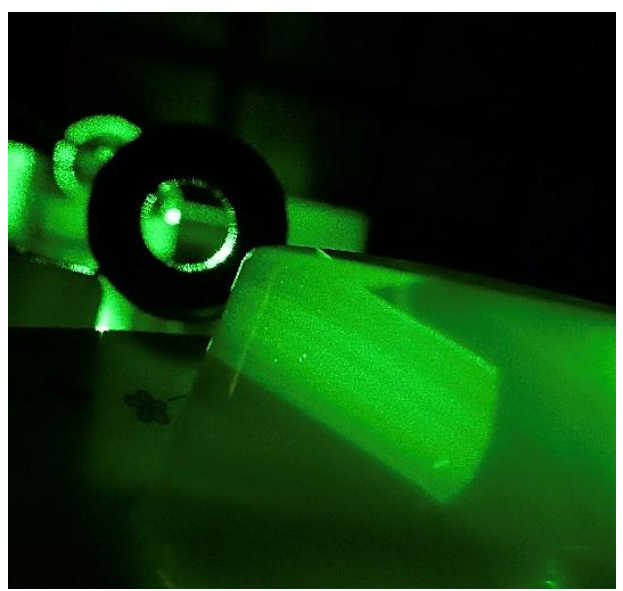

Figure 8. Discrete individual light strands created during diffraction viewed as striations while traversing gelatin media 

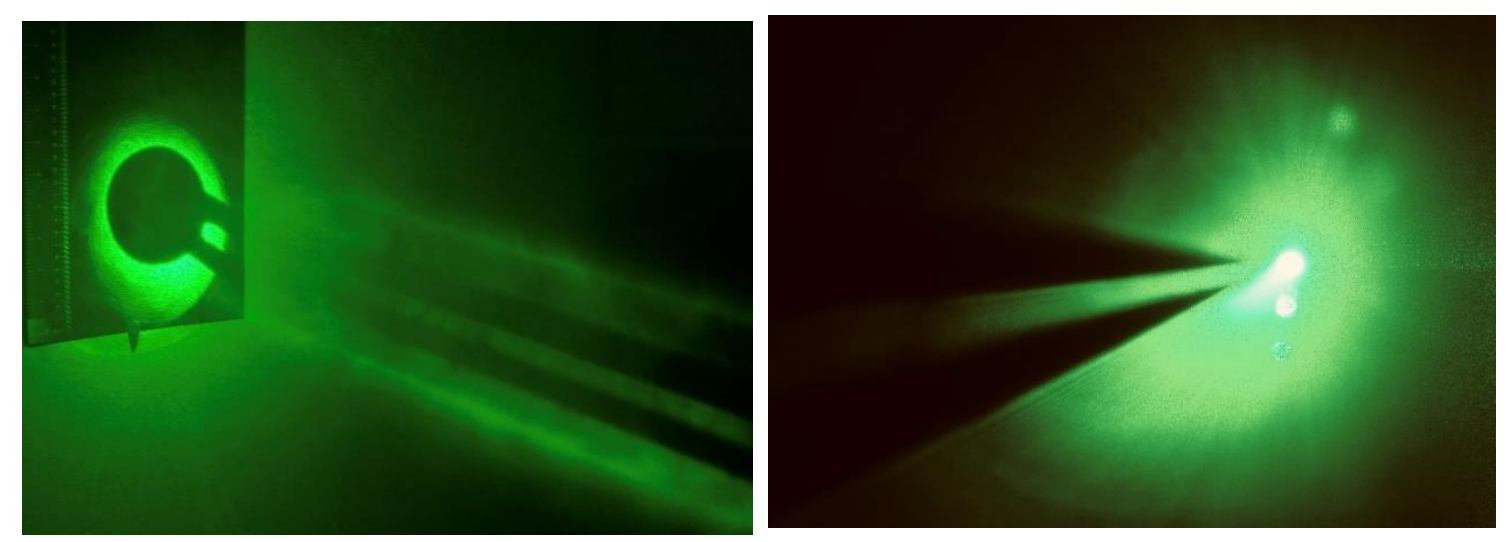

Figure 9. Cylindrical rim diffraction viewed externally and internally in a water vapor cloud chamber
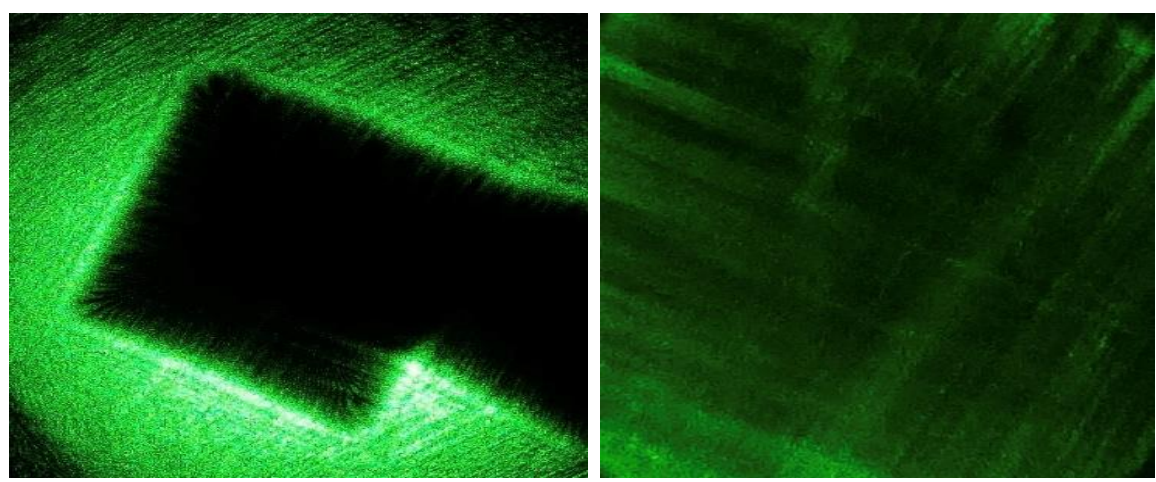

Figure 10. Square object reflecting light strands at right angles creating a hash pattern
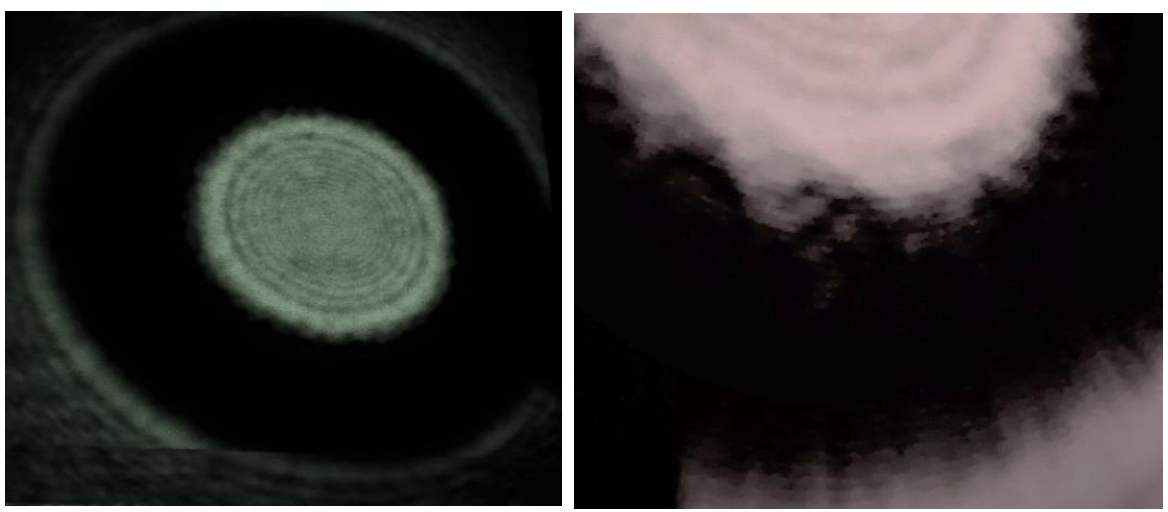

Figure 11. Solid ring object creating distinct concentric light rings from reflections away from rim edges of the shadow. In contrast, reflections inward to rim edges of the shadow are irregular not smooth showing partial linear and ring formations.

In the cloud chamber of water vapor, the cylindrical outline of the light radiation is noted (Figure 9). It is striated as it consists of individual light strands. The sharp border of two clear areas are visible traversing with the light strands and corresponding to the two jaws of the alligator clamp that blocked diffraction at the rim. Another view inside the projected light cylinder also shows the striated light strands around the periphery with distinct demarcation of the areas where the light is blocked by the clamp jaws. Faint strands are noted crossing towards the center creating the central spot.

Different shapes cause different reflection patterns. Square objects create lines that radiate perpendicularly from each edge (Figure 10). As there is no curvature, the lines never intersect at a central spot, but instead cross in a hash pattern.

Objects with central openings like rings and pinholes create light rings instead of lines (Figure 11). The rings correspond to the intensity of the light. The greater the intensity, the greater the visibility of rings. The smaller the aperture, at the same intensity of light, the more visible rings are internal to the rim edge as light reflections are more concentrated. The inward facing rims of the ring shadow are irregular, not smooth. These areas show partial wave and line formations. 


\section{Discussion}

Consistent with our observed findings, the Fresnel central spot can be explained by linear strands of light that radiate from the rim of the solid disc and intersect at the center of its shadow. Diffraction at one locus on the disc rim, perpendicularly reflects multiple strands with intervening clear spaces in a linear fashion, creating an image of a line on the screen (Figure 12). Diffraction at multiple loci on the rim for 360 degrees creates multiple lines. At the central point of intersecting lines, the illusion of a central spot is created (Figure 13). Wave concepts are not consistent with the observed distinct, linear images [12-14]. Wave interference, instead, predicts a central energy peak that drops off sharply in the periphery of the shadow, unlike what is noted here. Particle theory with reflecting light strands better explains the sharply demarcated lines throughout the shadow. The central spot ensues as these multiple low intensity strands intersect in the center of the shadow
Blockage at the rim of the disc creates a distinct blank demarcation in the lines crossing the shadow. This is consistent with light strands being finely blocked creating sharp demarcation in its radiations (Figure 14). Blockage of wave interference would not cause these sharp demarcations when obstructed and would only lessen central spot intensity.

The persistence of the light lines in the center of a ring obstruction, again argues in favor of particle theory (Figure 15, Blockage B). Particle theory can explain this as linear strands traversing through the center of the ring before striking the screen. Wave interference in this scenario would not show sharply delineated lines in the center of the ring shadow. It may even suggest the creation of a new central spot from new emanating waves from the edges of the ring, unlike what was observed.

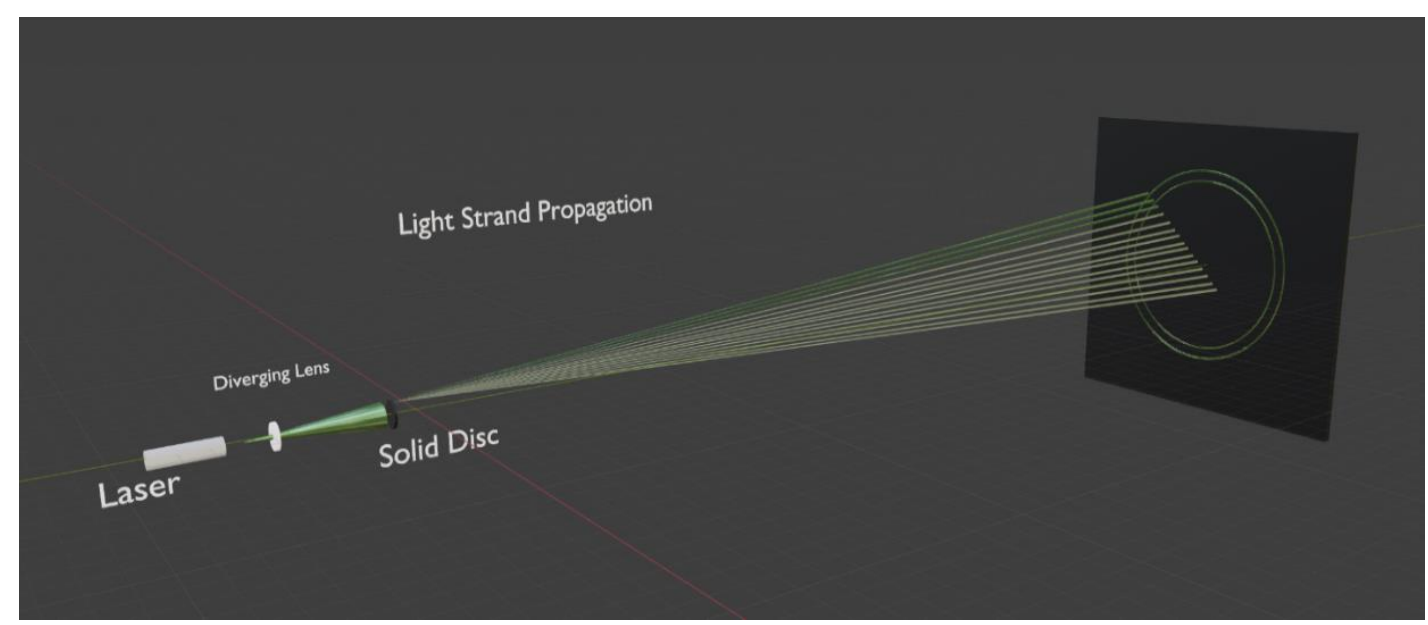

Figure 12. Light strands radiating from one locus of disc rim and forming a line pattern on the shadow.

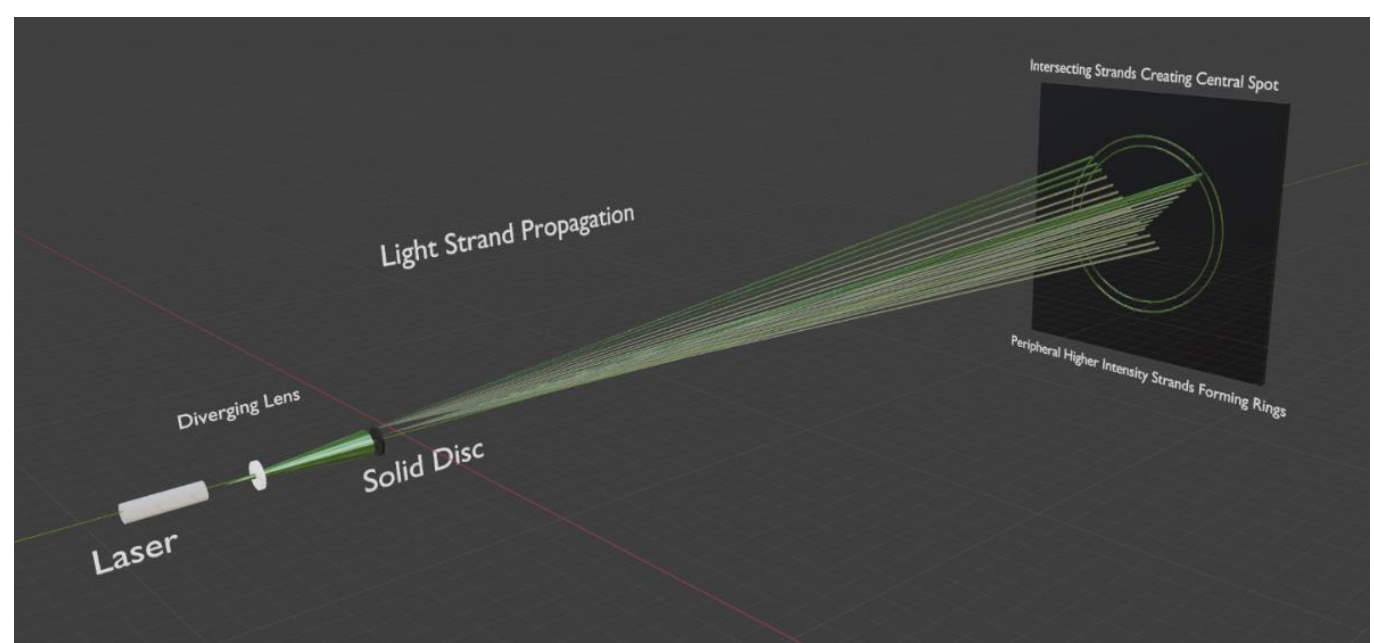

Figure 13. Multiple projected lines of light intersecting in center of shadow creating a bright spot. Peripheral higher intensity strands that are adjacent merge to form rings. 


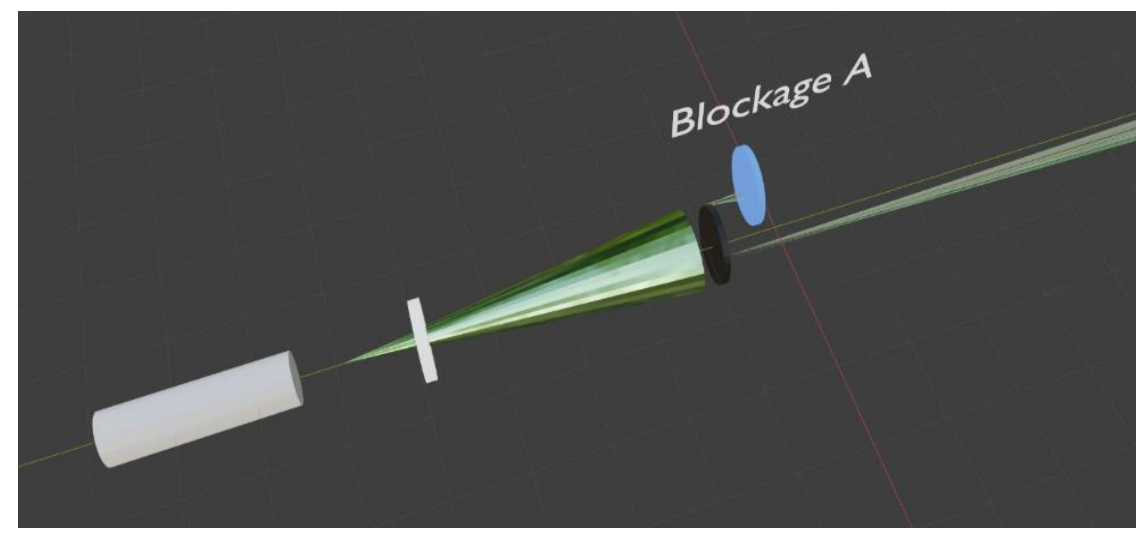

Figure 14. Blocking light at the point of diffraction (Blockage A) removes the entire line of radiating strands creating a finely demarcated clear space

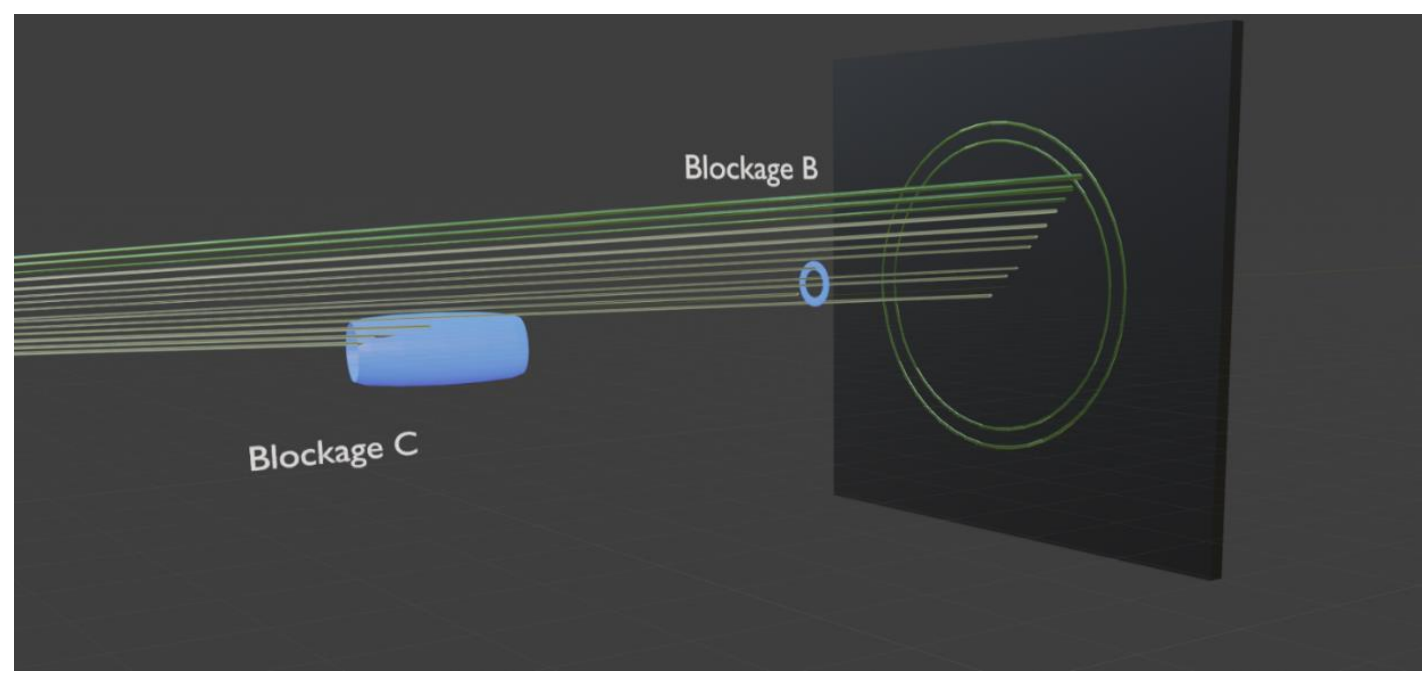

Figure 15. Light blockage with ring (Blockage B) showing transmission of central light strands and tube (Blockage C) showing obstruction of central light strands

A hollow tube placed perpendicularly to the screen, should allow light to pass through if light waves or particles were radiating perpendicularly from the disc. This was not observed, as the shadow was solid when a tube was placed in front of the screen. This observation is more consistent with angled radiating strands being blocked by the perpendicular tube as they try to cross through the center of the shadow (Figure 15, Blockage C).

Illuminating only a locus on the disc rim only creates lines from that spot. This is consistent with light strands only emanating from that point. These lines also show a fringe pattern. This is consistent with prior observations of fringe patterns when light reflects off edges [11]. This supports the conjecture of the central spot as the confluence of reflections off multiple loci on a circular disc. The reflecting strands intersect in the center of the shadow creating the pseudo-spot.

The reflections consist of an alternating sequence of bright and clear spaces (Figure 12). The bright space corresponds to the strand width (M) and the clear space to the intervening clear space width (I) between strands. Each pair width $(P)$ of strand and clear space at a set intensity can be described as (Equation 1):

$$
M+I=P
$$

From previous analysis [11], using a $5 \mathrm{mw}$ $532 \mathrm{~nm}$ laser, we can determine the size of each component at a distance $(x)$ from the diffraction source (Equation 2).

$$
.0016 x+.00027 x=P
$$

The radius $(r)$ of a shadow at distance $x$, allows calculation of the number of pairs to reach the center (Equation 3).

$$
r / P=n_{\text {pairs }}
$$

Observations with the solid square object further support the reflecting strands concept. Lines radiating perpendicular to the edge in a linear path create discrete lines in a hash pattern (Figure 16), unlike wave interference expected results.

The periphery of the shadow shows a tendency to form rings (Figure 13). It appears to be dependent upon the amount of photon energy available. 


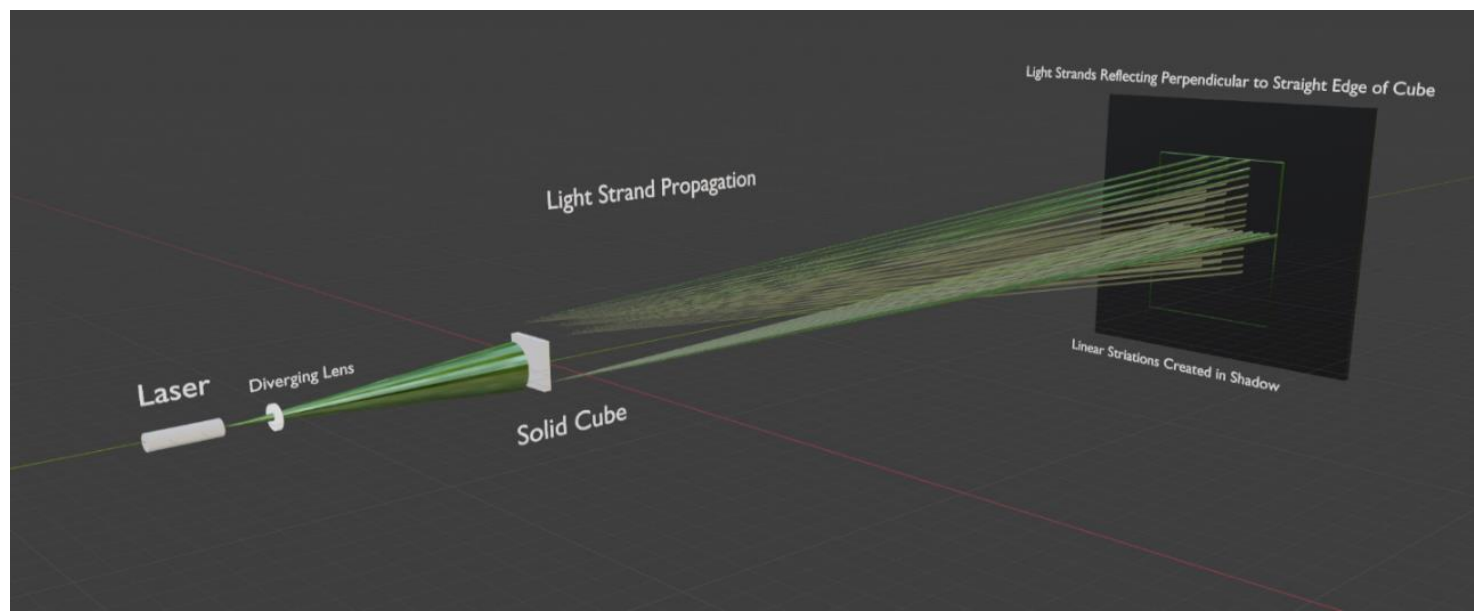

Figure 16. Light strands diffracting perpendicularly off a square object and creating linear hash marks on the screen

Reflections external to the shadow rim are of high intensity and form sharp rings. Reflections internal to the rim are of low intensity and form lines with fringe patterns. But at the internal edge of the rim where the relative light intensity is intermediate, irregular patterns are formed. Observing rings and pinholes with smaller central apertures, where more light energy reflects inward from the inner rim, complete rings are noted (Figure 11). Here also along the inward shadow borders, where there are fewer reflecting light strands, an irregular border appears where partial lines alternate with partial rings. An explanation to satisfy these findings is failed ring formations due to insufficient energy. A photon energy threshold $\left(E_{\min }\right)$ appears to determine whether strands stay discrete and form lines with fringes $\left(E_{1}\right)$ or fuse with adjacent strands to form intermittent rings $\left(E_{2}\right)$. This quantized behavior can be described with equations of energy levels (Equation 4: $h$ - Planck's constant, $c$ speed of light, $\lambda$ - wavelength):

$$
\begin{gathered}
E_{1} \rightarrow E_{2} \\
E_{1}+E_{\text {min }}=E_{2} \\
E_{\text {min }}=n_{\text {photons } / \text { strand }} \times n_{\text {strands }} \times h c / \lambda
\end{gathered}
$$

To reach the higher energy state, either the photon density or the strand density must increase. At this higher energy level, strands appear to merge with adjacent strands to form a ring while the clear intervals of its fringe pattern remain to separate the confluent rings. Our previous equation (Equation 3 ) of strand and clear interval pairs ( $\mathrm{r} / \mathrm{P}=\mathrm{n}$ pairs) can therefore also represent the number of potential rings within a shadow of radius $r$.

\section{Conclusion}

The Fresnel central spot can be explained by particle theory. Linear light strands, not wave interference, reflecting perpendicularly off the edge of the circular disc and intersecting as they cross the center of the shadow create the appearance of a pseudo-spot.
The central spot has traditionally been viewed as strongly supporting wave concepts of light, but this may be erroneous. Further evaluation may be helpful to determine if linear light strands explain observations of light behavior better than wave theory in other situations. Adjacent light strands also appear to merge forming rings contingent upon a photonic energy threshold. This appears to be another quantized behavior of light.

\section{References}

[1] K.F. Kuhn, F. Noschese, (2020) Light: Wave or Particle? Basic Physics, Wiley, New Jersey, 227-236.

[2] G. Carroll, (2020) Quantum Physics for Beginners, Carroll, 46-112.

[3] C.J. Pratt, (2021) Quantum Physics for Beginners: From Wave Theory to Quantum Computing, Stefano Solimito.

[4] S. Bembenek, (2017) The Cosmic Machine: The Science That Runs Our Universe and the Story Behind It Kindle Edition, Zoari Press.

[5] Wave-Particle Duality in Quantum Physics for Beginners: All the Major Ideas of Quantum Mechanics, Modern Science, 2021, 18-20.

[6] J. Law, R. Rennie, (2015) A Dictionary of Physics, Oxford University Press. [DOI]

[7] F.L. Pedrotti, L.S. Pedrotti, L.M. Pedrotti, (2007) Introduction to Optics, Cambridge University Press.

[8] J. Walker, D. Halliday, R. Resnick, (2014) Halliday \& Resnick Fundamentals of physics, Wiley, New Jersey.

[9] Augustin Jean Fresnel, Henry Hureau de Sénarmont, Émile Verdet, L'eonor François Fresnel, (1866) Complete Works of Augustin 
Fresnel: Theory of Light, Imperial printing press.

[10] D. Arago, Report made by Mr. Arago to the Academy of Sciences in the name of the commission which had been charged with examining the memoirs submitted to the competition for the diffraction prize, Annales de Chimie et de Physique, 11 (1819) 5-30.

[11] S. Dutt, N. Dutt, A. Dutt, Light Strands: Visualization of Free Space in Double Slit Diffraction, International Journal of Physics, 9 (2021)197-205. [DOI]

[12] T. Reisinger, P. Leufke, H. Gleiter, H. Hahn, On the relative intensity of Poisson's spot, New Journal of Physics, 19 (2017) 1-16 [DOI]

[13] M. Born, E. Wolf, (1999) Principles of Optics, Cambridge University Press. [DOl]

[14] C.R. Nave, (2017) Hyperphysics, Hyperphysics. Department of Physics and Astronomy, Georgia State University, Atlanta.

Conflict of interest

The Author has no conflicts of interest to declare that they are relevant to the content of this article.

Does this article screened for similarity?

Yes

About the License

(C) The Author 2022. The text of this article is open access and licensed under a Creative Commons Attribution 4.0 International License. 